\title{
High Precision Temperature Control System for an Oven-Controlled Crystal Oscillator
}

\author{
Dan Xie ${ }^{1,2}$, Xuefeng Chang ${ }^{1,2, *}$, Xiayun $\mathrm{Shu}^{1,2}, \mathrm{Li} \mathrm{Li}^{2}$, Jian Wang ${ }^{1,2}$, Lifang Mei ${ }^{1,2}$ and Yangxue \\ $\mathrm{Liu}^{1,2}$ \\ ${ }^{I}$ School of Mechanical and Automotive Engineering, Xiamen University of Technology, Xiamen, 361024, China \\ ${ }^{2}$ Key Laboratory of Precision Actuation and Transmission, Fujian Province University, Xiamen, 361024, China
}

\begin{abstract}
Environmental temperature is a highly influential factor regarding the stability of the crystal oscillator frequency. Thus, a high precision temperature control system is critical for improving the frequency stabilization of crystal oscillators. This paper proposes a temperature control system, including a high sensitive thermistor, precision reference voltage source and an instrumentation amplifier for the temperature measurement and preamplifier. System level MCUMSC1210 acts as a main controller, and a novel way of the "interrupt + ribbon" strategy is adopted to realize real-time task scheduling. The PID controller program is crafted with the help of the "anti-integral saturated" and "bumpless transfer" algorithms. The cascade control system with the heating current of negative feedback is then realized using the triode tube consumption heating style. The results show that the temperature and fluctuation of heat voltage have almost no effect on the heating current when the heating system voltage fluctuates between $2.2 \sim 8 \mathrm{~V}$. Within those parameters, the temperature deviation remains within 0.01 degrees. Thus it can be seen that the high-performance temperature control system has a high anti-interference capability and a similarly high level of accuracy.
\end{abstract}

Keywords: Oven controlled crystal oscillator, precise temperature control, pulse width modulation, embedded system.

\section{INTRODUCTION}

The Crystal Oscillator acts as the frequency generator with high precision and stability in all kinds of oscillation circuits and communication systems. Known as the "heart" of electronic systems [1-4], the Crystal Oscillator outputs a high-precision signal, regarded as the time benchmark and reference frequency. The utility of Crystal Oscillators is widely applied in various areas, including: communication base stations, guidance systems, precision metering, radar, satellite, global positioning, atomic clocks, telemetry and telecontrol. The quality and stability of the Crystal Oscillator's output signal by are major factors in determining the quality of an electronic system. But since Crystal Oscillator usually works in a very complicated environment, a number of phenomena, such as temperature field, electric field, magnetic field and vibration, have various effects on the output frequency signal. The Oven-Controlled Crystal Oscillator (OCXO) is a type of crystal oscillator that keeps the temperature of the quartz crystal resonators stable by means of thermostat, thus minimizing the change in output frequency triggered by temperature variation. Therefore, a highprecision temperature control system plays a key role in increasing the frequency stability of the OCXO [5-9].
The design and development of a high-precision temperature control system in OCXOs has aroused public concern from scholars both at home and abroad. As for overseas studies, Russian researchers took advantage of new technologies that increase the thermostat baths and reduce the outer temperature gradients. Using this method, the researchers then developed a temperature-controlled high stability crystal oscillator with pony size and double layers. A frequency temperature stability of $\pm 1 \times 10$ - 10 could be achieved within the range of -40 to $+70{ }^{\circ} \mathrm{C}[10]$. In another study, which was completed in Japan, Nihon Dempa Kogyo Co., Ltd. measured the temperature based on the difference in the frequency between the vibration of Parylene B and that of Parylene C. by outputting the vibration of Parylene $\mathrm{C}$ from the clock in use. The crystal temperature remained locked within \pm $0.002{ }^{\circ} \mathrm{C}$ when ranging from -20 to $+70{ }^{\circ} \mathrm{C}$ [11]. In contrast, domestic research began relatively late, and has mainly been conducted from within scientific research institutions, colleges and universities. Recently, however, a series of achievements have been made through a series of studies concerning high stability OCXOs by a number of institutions, including: No. 18 and No. 27 Research Institutes of CETC (China Electronics Technology Group Corporation), No. 203 Research Institute of CASIC (China Aerospace Science and Industry Corporation), HUST (Huazhong University of Science and Technology) and Xidian University [12-14].

Both the software and hardware used in the high-quality precise temperature control system of the quartz crystal oscillator were designed in this work. The study used a highly 


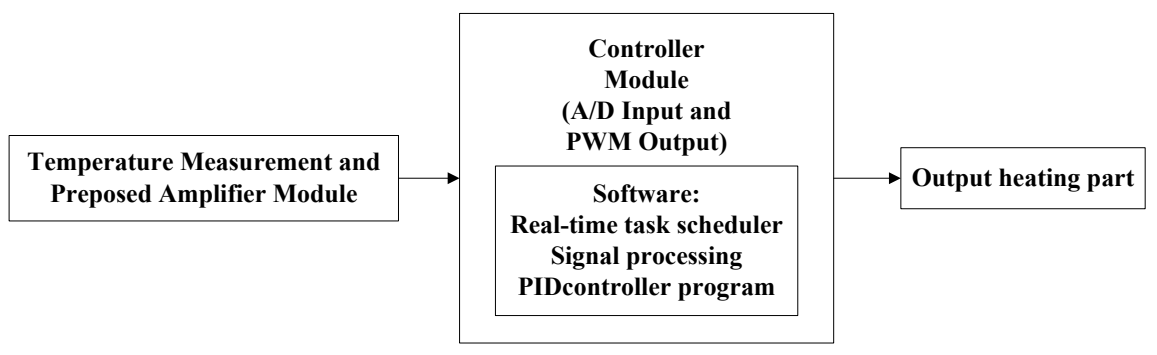

Fig. (1). System Function Module.

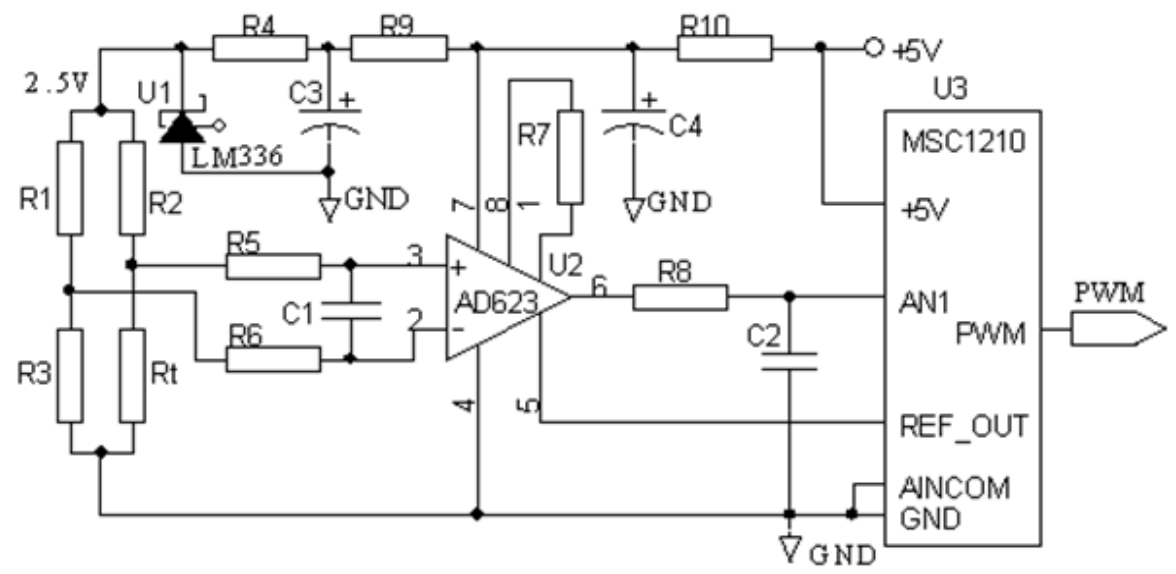

Fig. (2). Temperature Measurement and Preposed Amplifier Circuit.

sensitive thermistor and precision reference voltage source preposed with an instrumentation amplifier, which was composed by its temperature measuring and the magnified part. After amplifying the bridge's voltage signal to help in measuring the temperature, the output PWM control signal was processed. Then, the current was heated through the use of precise filtration and Voltage/Current transfer control. Consequently, the margin of error regarding the steady-state temperature of the crystal oscillator was controlled within \pm $0.01{ }^{\circ} \mathrm{C}$, thus achieving the purpose of precision temperature control.

\section{SYSTEM HARDWARE AND SOFTWARE DESIGN}

\subsection{System Function Module}

This work designed a temperature control system comprised of a high quality quartz OCXO complete with a hardware system including a controller module, temperature measurement, preposed amplifier module and output heating module. The software system included a task dispatcher, digital signal processing program and PID controller (See Fig. 1).

\subsection{Temperature Measurement and the Preposed Ampli- fier Module}

The temperature detection module is a key factor affecting the quality of high-precision temperature control systems.
The NTCR (negative temperature coefficient resistance), which served as the temperature sensor, is small in size and light in mass so that the accuracy of the temperature measuring bridge is high. Fig. (2) shows a detailed circuit diagram as well as the output voltage of the thermistor bridge.

$$
V_{t}=U\left(\frac{P_{t}}{R_{2}+P_{t}}-\frac{R_{3}}{R_{1}+R_{3}}\right)
$$

When $V_{t}$ reaches the maximum at the constant temperature, the measurement module will produce the highest sensitivity.

$U\left(\frac{P_{t}}{\partial R_{2}+P_{t}}-\frac{\partial R_{3}}{\partial R_{1}+\partial R_{3}}\right)=0$

According to Formulation 2 above, the electric bridge can get the best sensitivity when the value of arm resistance equals the value of thermistor at the required constant temperature.

The measurement accuracy of the electric bridge is greatly affected by the stability of precision electric bridge service voltage. Accordingly, the precision benchmark power of LM336 and a dynamic resistance with $0.2 \Omega$ should be selected, to be consistent with the precision electric bridge. With a pin of LM336, the reference voltage can match the temperature coefficient, and the stable reference voltage of $2.5 \mathrm{~V}$ can also be elicited expediently from the $5 \mathrm{~V}$ power supply. In order to avoid outside interference to the small 


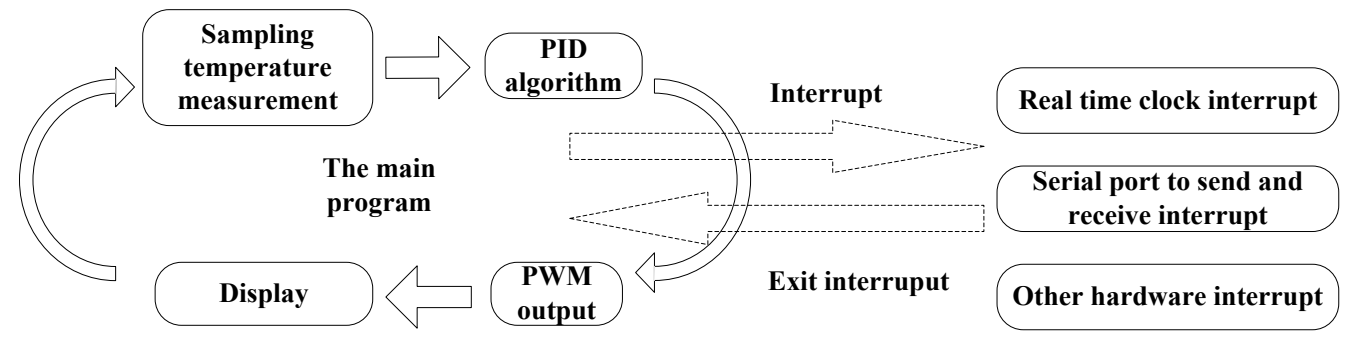

Fig. (3). Real-Time Scheduling Program Flow.

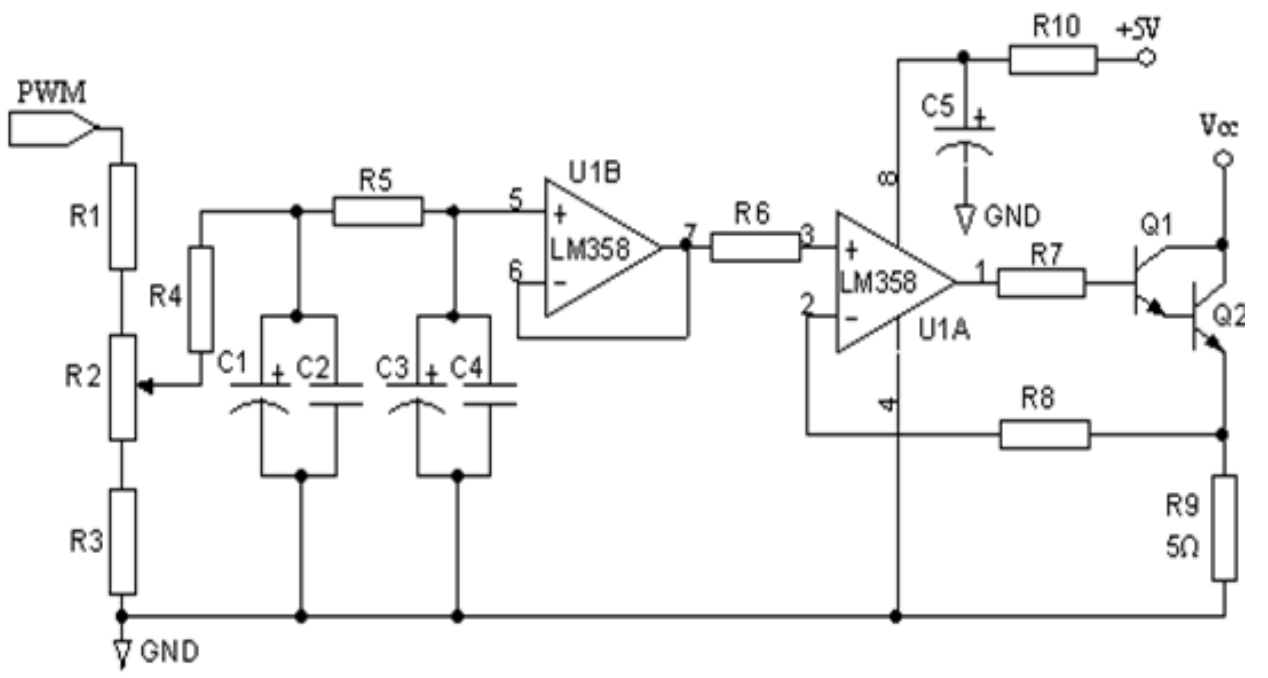

Fig. (4). Precise Filtration, Voltage/Current Convert, Current Drive and Tube Heating Circuit.

signals generated by the temperature measurement bridge, an amplifier chip, AD623, can be added as a preposed instrumentation amplifier. Due to the amplifier's fine linearity, stable temperature and reliability, a gain coefficient from 1 to 1,000 can be obtained in coordination with the external resistance. In the meantime, the AD623 acquires a strong degree of anti-common mode interference, through laser retouching in the manufacturing process, which effectively enhances the system's accuracy and anti-interference ability.

\subsection{Controller Module}

The temperature control system consists of the singlechip system MSC1210. This one chip integrates the A/D converter and its reference voltage, microprocessor and PWM output. In this way, only a small amount of hardware systems are needed to do the heating and temperature measurement, which greatly simplifies the external circuit design.

This work applies real-time multitask scheduling, achieved by use of the "interruption plus round-ribbon" strategy. Temperature measurement sampling, PID operation and PWM signal output are set in the main program to form a loop body. After that, high-level real-time tasks, such as real-time clock and serial transceiver programs, are processed by means of an interrupt handler. Fig. (3) shows the program flow.

\subsection{Output Heating Module}

\subsubsection{Output Heating Circuit}

Using Audion tube heating, the control signal of the output heated part can generate heat to the Audion through precise filtration, voltage/current conversion, and a Darlington current drive circuit (See Fig. 4).

Despite this, the Audion tube heating mode has a high degree of universality. The control circuit accuracy is largely affected by the nonlinear relationship between the heated current through the Audion and PWM duty cycle. This work completed the linear conversion of voltage/current by implementing the cascade control scheme. With a strong antiinterference ability, it can adjust the temperature parameters effectively, which otherwise lags too much. Thus, using this scheme may help overcome the impact of heated currents on its temperature.

\subsubsection{Cascade Control System}

In this cascade control system, the control circuit is composed of a temperature regulation system, which allows the researcher to maintain a high degree of control over the accuracy and negative feedback of the heated current. The temperature regulator in the adjusting loop can control the 


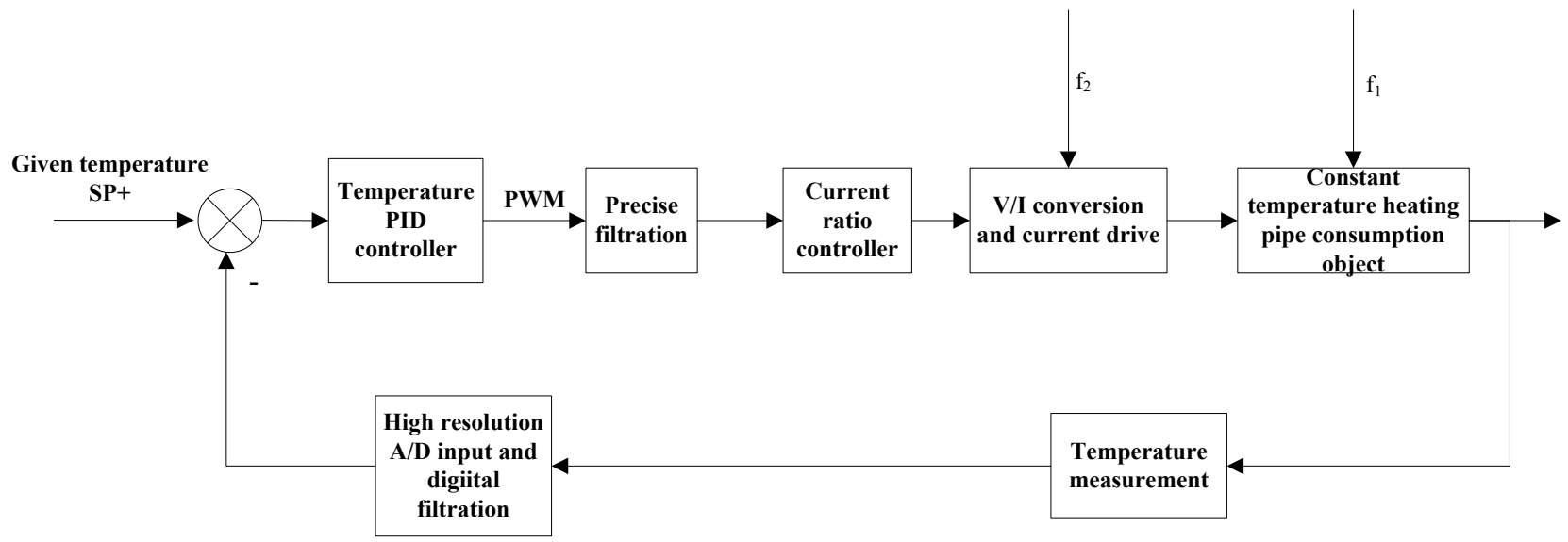

Fig. (5). Temperature Regulation System.

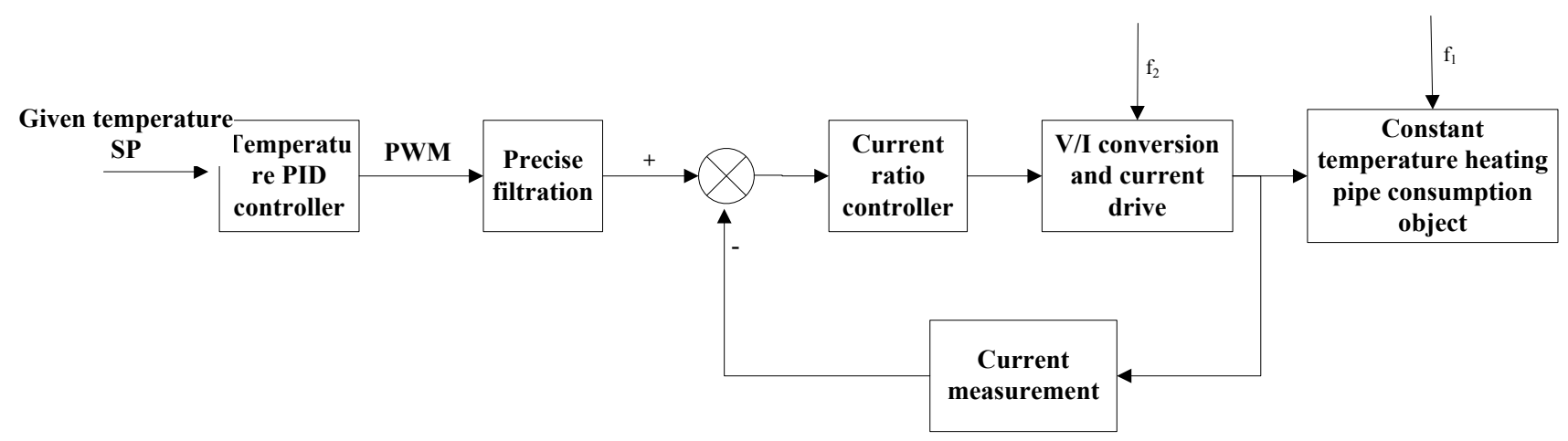

Fig. (6). Block Diagram of Single-Ring Current Control System.

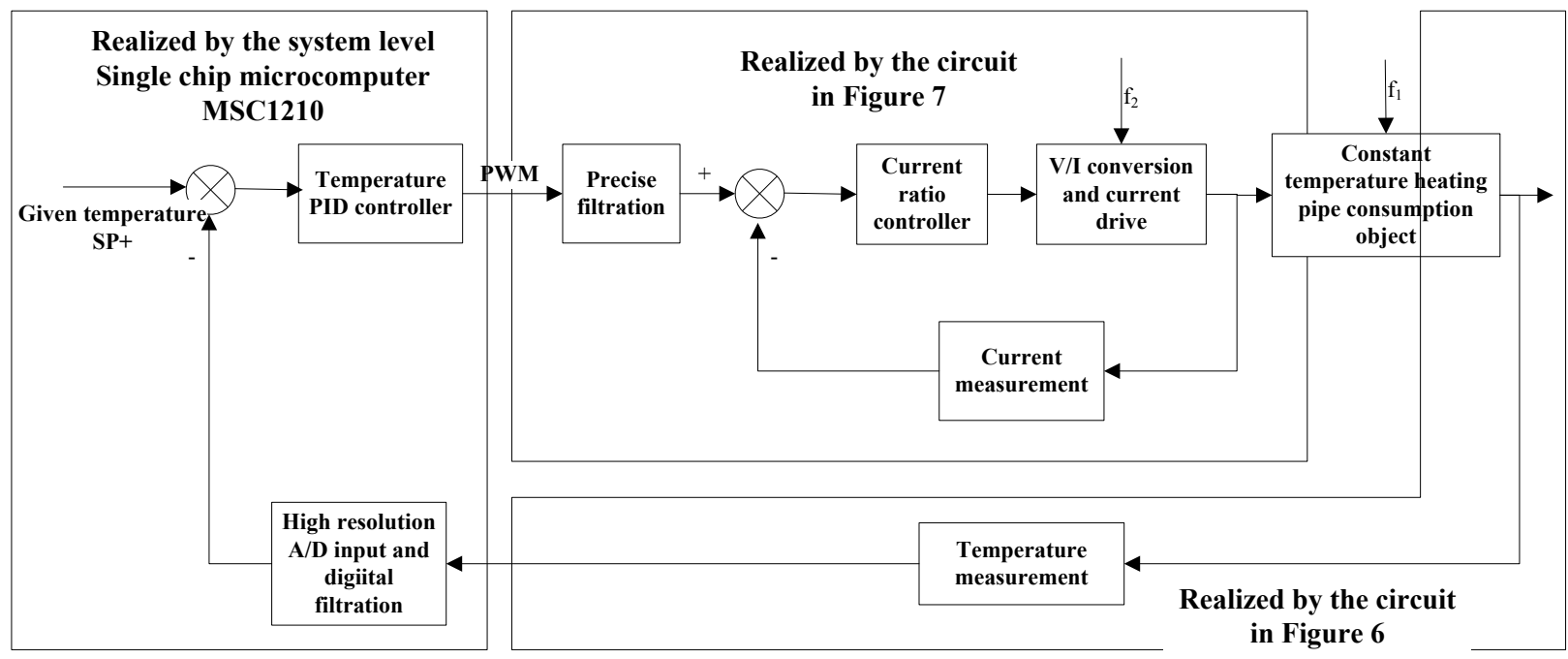

Fig. (7). Block Diagram of Cascade Control.

PWM output duty cycle and eliminate the external interference. However, a slight disturbance in this current can cause fluctuation in the temperature regulation. Since the control circuit is composed of the negative feedback taken from the heated current, it can quickly surmount any interference in the heated current and eliminate poor linearity; in addition, it can disperse the device parameter in tube heating mode, as well. Therefore, the temperature regulation system and the heated current adjusting system should be concatenated together, so as to create a cascade control system, with both internal and external loops (See Fig. 5-7).

An output heated current, through resistance R8, can introduce the negative feedback of the current to the negative input end of the operational amplifier from the current meas- 


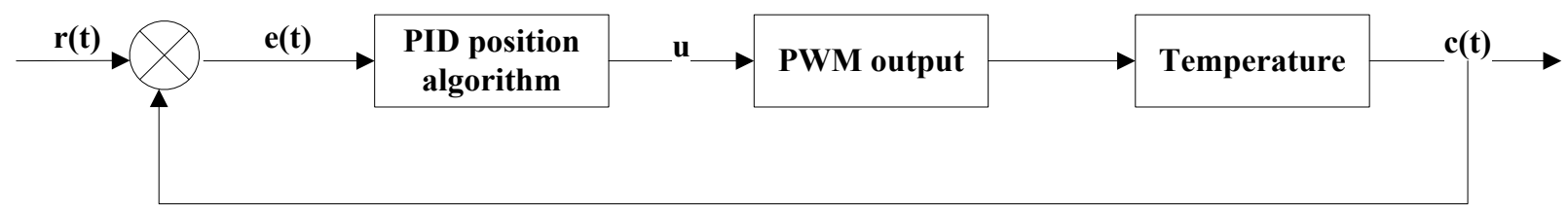

Fig. (8). PID Control Circuit.

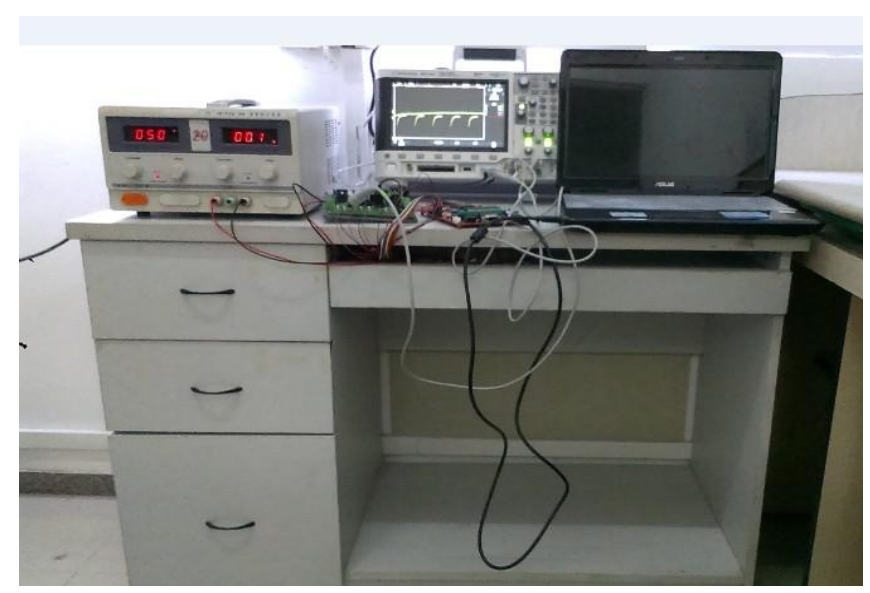

Fig. (9). Experiment Device.

uring resistance $\mathrm{R} 9$. Thus, an internal circuit that contains a cascade control loop and a current regulator is then connected, thereby endowing the whole control system with a strong anti-interference ability.

\subsection{PID Controller Design}

A PID controller is used to gather feedback from the control circuit. With the formation of the PID control circuit, we used a positional PID algorithm to calculate the PWM duty cycle. In this manner, the heating power of the thermostat can be controlled. Fig. (8) shows the control circuit, wherein the detailed methods are as follows.

In the digital control system, a discrete control system can emerge with such a mode, where the single-chip takes advantage of the equal time interval $(\triangle T)$ to process samples and the PID control. The following is the difference equation that is used in the PID control.

$e_{k}=r_{k}-c_{k}$

$u_{k}=K_{p} \cdot e_{k}+\left(K_{p} \cdot \frac{\Delta T}{T_{i}}\right) \sum e_{j}+\left(K_{p} \cdot \frac{\Delta T}{T_{i}}\right) e_{k}+\left(K_{p} \cdot \frac{T_{d}}{\Delta T}\right) e_{k},\left(e_{j}=0,1, \ldots k-1\right)$

where point $r_{k}$ is the set value; $c_{k}$ is the process variable; and the $e_{k}$ error is the value in the $k$ times sampling.

The difference of error value between this time and the last time is $\Delta e_{k}=e_{k}-e_{k-l}$. Therefore, $\Delta T$ is the sampling period using the units of measurement $\mathrm{ms}$ or s. In order to improve the calculation speed of the programming, Equation 4 can be improved as :

$u_{k}=K_{p}\left[e_{k}+\left(\frac{1}{T_{i}}\right) \sum e_{j} \cdot \Delta T+T_{d}\left(\frac{\Delta e_{k}}{\Delta T}\right)\right],\left(e_{j}=0,1, \ldots k\right)$
$u_{k}=K_{p} \cdot e_{k}+A+B \cdot e_{k}+C \cdot \Delta e_{k}$

where

$$
\begin{aligned}
& A=(K p \cdot \Delta T / T i) \sum e j,(e j=0,1, \ldots k-1) \\
& B=K p \cdot \Delta T / T i \\
& C=K p \cdot T d / \Delta T
\end{aligned}
$$

In the initialization and PID reference shift program, when $\mathrm{A}=0$, the values $\mathrm{B}$ and $\mathrm{C}$ can be calculated according to Equation (8) and Equation (9). Following three multiplications, three additions, and no division, Equation (5) can be computed in every sampling, which can drastically reduce the calculation quantity and enhance the computing speed of the controller program. Programming the PID controller in such a way can improve the algorithm of the computing speed, and the algorithms of the anti-windup and the bumpless transfer can then be added, based on the requirements for industrial application.

\section{EXPERIMENT AND RESULT ANALYSIS}

Fig. (9) shows the self-designed experiment system, including the heating and temperature measurement circuit module, the precise filtration circuit module, and the voltagestabilizing circuit module for the bridge. Negative feedback from the heated current is introduced to form an internal loop for the cascade control circuit. The control circuit forms after using resistance to check the heated current and providing the feedback from the voltage from both the ends to the negative input end of the operation amplifier in this level.

Firstly, according to Fig. (2) of the cascade control circuit, the external ring is disconnected. The internal ring is used to 


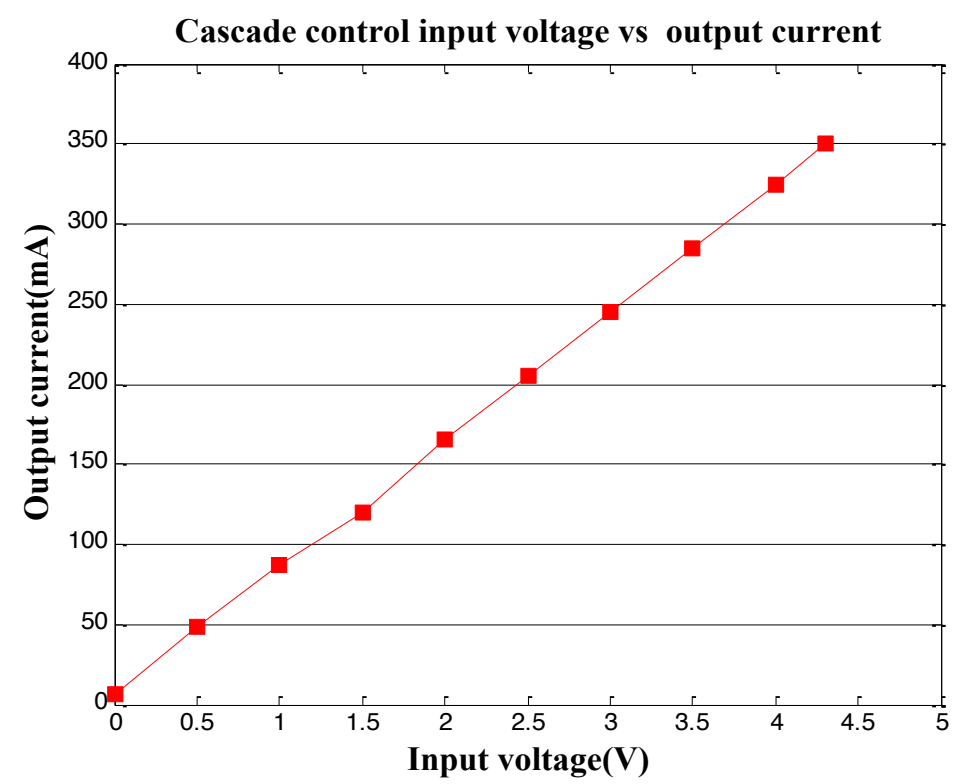

Fig. (10). Voltage/Current Convert Experiment Curve.

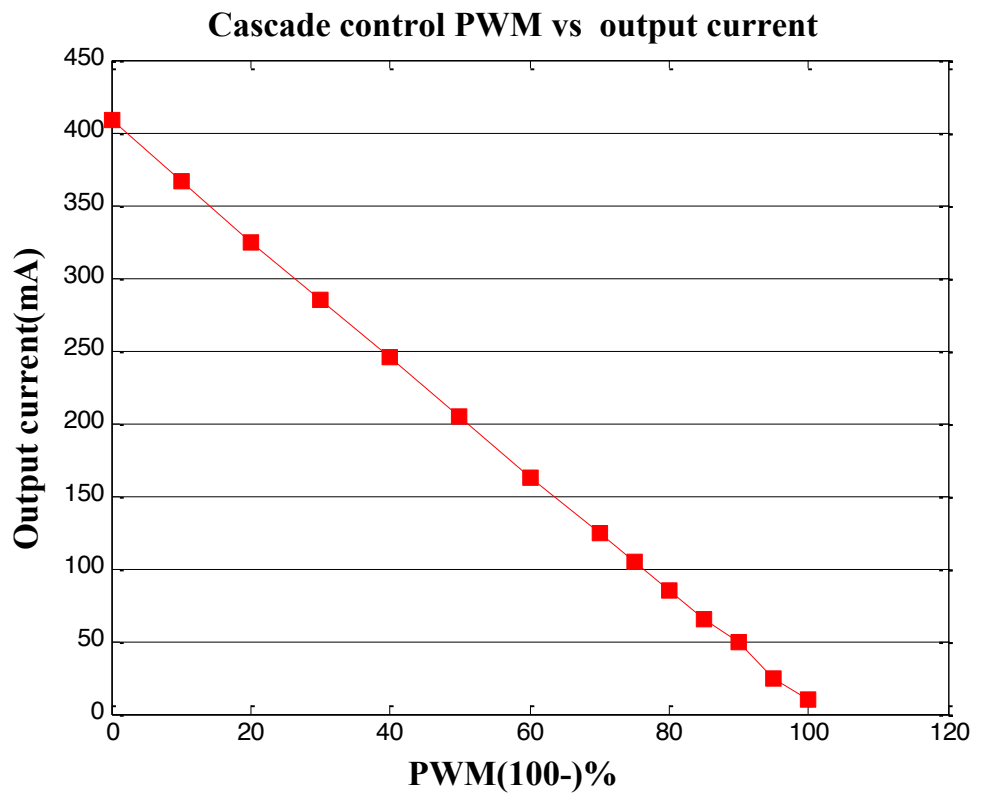

Fig. (11). PWM/Current Convert Experiment Curve.

maintain control, and the power $\mathrm{Vcc}$ is adjusted to $5 \mathrm{~V}$. As shown in Fig. (4), the DC voltage and PWM percentage signal are separately input in the PWM output end, and then converted into an empirical curve within the tube heating current. The available experiment results are displayed in Fig. (10) and Fig. (11), which indicate a fine linearity relationship between both the input and the output.

Then, based on the circuit displayed in Fig. (2), we can acquire the influence curve of the tube heating current, by accounting for the heated voltage Vcc, after inputting the PWM fixed percentage signal from the PWM end (See Fig. 12).
It can be concluded that this circuit has a strong antiinterference ability. When heating voltage Vcc fluctuates in the range from 2.2 to $8 \mathrm{~V}$, the output current value is very stable, nearly undisturbed by the voltage fluctuation. As seen in Fig. (4), the fluctuation of the heating voltage is equivalent to the internal interference $f 2$ in the cascade control circuit, the jamming effects of which are suppressed and offset under the help of an internal-ring control. As a result, we have minimized the impact of the fluctuations of the heating voltage on the external ring. Since the external ring is mainly used to suppress and offset the impact by interference f1, the cascade control can largely enhance the system quality and accuracy. 


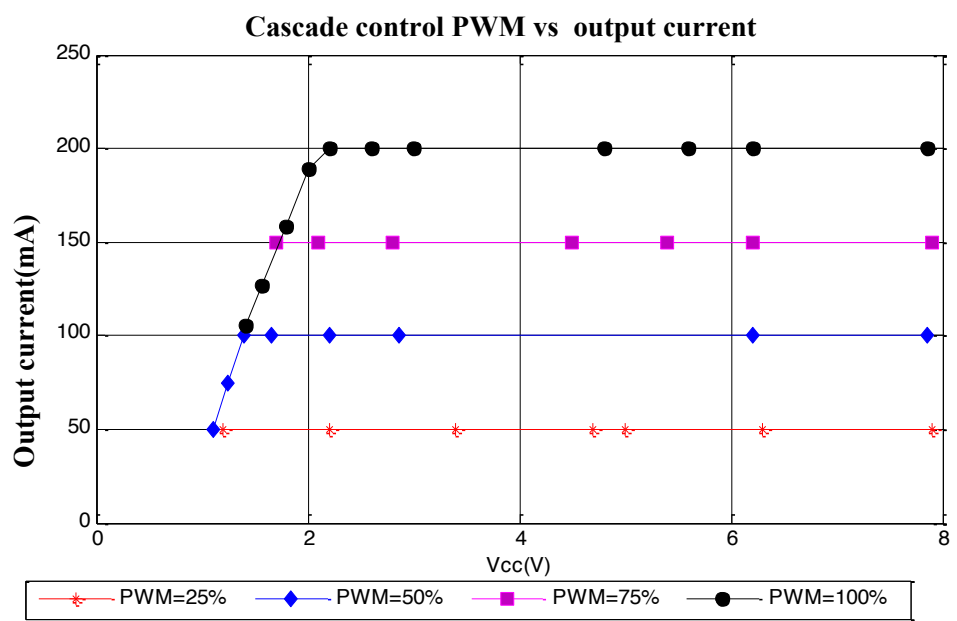

Fig. (12). Experiment Curve of Anti-Heating Voltage Fluctuation.

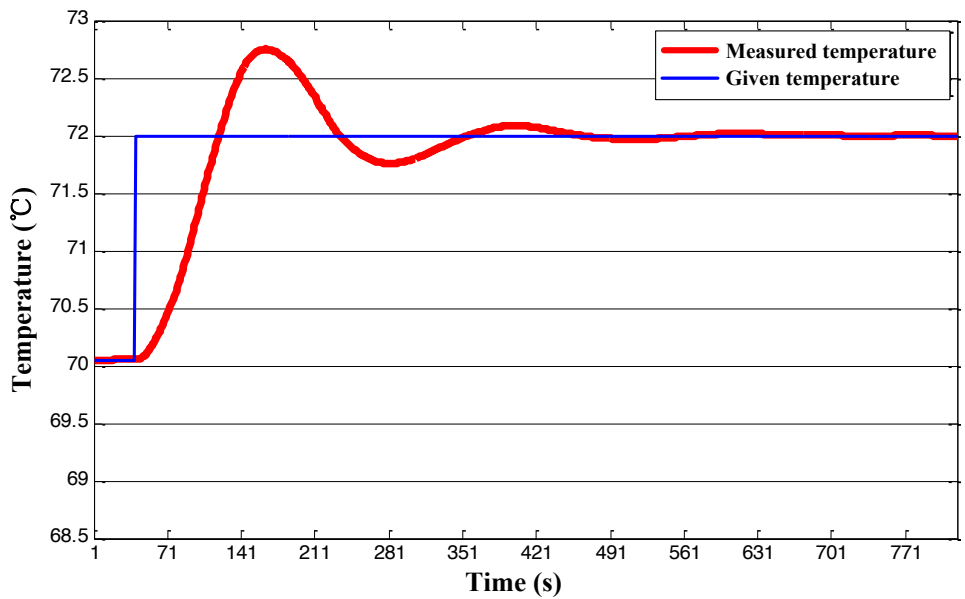

Fig. (13). Experiment Curve of Dynamic Performance.

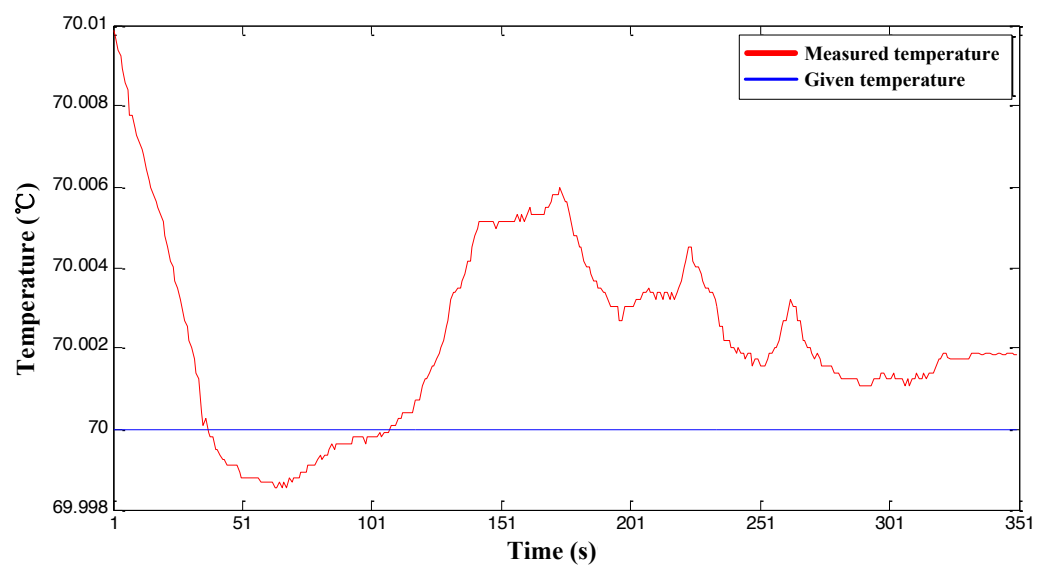

Fig. (14). Experiment Curve of Static Error.

Based on the data from the quality test of the temperature control system, as in Fig. (13) and Fig. (14), it is evident that the cascade control system has a good dynamic and static performance, whose control accuracy can amount to \pm $0.01{ }^{\circ} \mathrm{C}$.

\section{CONCLUSION}

This work explored temperature measurement and heating by utilizing a temperature control system composed of system-level single-chip MSC1210. In addition, we decreased the amount of hardware needed for the temperature 
control system and effectively simplified the outer circuit. Using a highly sensitive NTC thermistor, precision reference voltage, and instrumentation amplifier, we suggest utilizing the aforementioned temperature measuring process and proposed magnified parts to improve the anti-interference ability of a temperature control system. The negative feedback provided by the cascade control system of the heating current is designed to eliminate the poor linearity and dispersion of the device parameter in the tube heating mode, and as enhance the anti-interference ability and control accuracy of the temperature control system. The "interruption plus round-ribbon" strategy is applied in order to achieve a realtime multi-tasking schedule. Compared with the common real-time operation system, the non-redundant temperature control system has better flexibility and reliability. By adding the algorithms of the anti-windup and bump-less transfer, we programmed the PID controller, thereby strengthening the utility functions of the PID controller. In conclusion, the system displays effective dynamic and static performance, with an accuracy error within the range of $\pm 0.01{ }^{\circ} \mathrm{C}$, so it is likely to create a temperature control system within a quartz crystal oscillator with a high degree of stability and precision.

\section{CONFLICT OF INTEREST}

The authors confirm that this article content has no conflict of interest.

\section{ACKNOWLEDGEMENTS}

This work was supported by the National Natural Science Foundation of China (Grant No. 51475400, Grant No. 51105321 and Grant No. 51405411), the Natural Science Foundation of Fujian Province (Grant No. 2013J05084) and the Xiamen Municipal Science and Technology Plan Project (Grant No. 3502Z20143032).

\section{REFERENCES}

[1] H.B.Zhu, Z.B.Wu, and G.Q.Liu. "Study of quartz temperature characteristics for precise oscillator applicationsr", Acta. Phys. SinCh Ed, vol. 62, no. 014205-1, 2013.

[2] S.Qin, Y.Gong, and W.Q.Yuan. "High precision temperature control for projection lens with long time thermal response constant," Opt. Precis. Eng, vol. 21, pp. 108-114, 2013

[3] L.Z.Ji, Q.X.Shan, and Q.Tang, "The acceleration effects on crystal oscillators and research on compensating technique", J. Astronaut. Metrol. Meas, vol. 32, pp. 37-42, 2012

[4] M.Q.Li, X.Lu, and R.H.Zhao, "Design of an ASIC for oven control crystal oscillator," Semicond Tech, vol 32, pp.832-835, 2012.

[5] H.H.Xie, W.D.Zou, and C.H.Huang, "LD temperature control system based on PWM comparison amplifier," Chinese.J.Sci.Instrum, vol. 30, pp. 1530-1534, 2009.

[6] Y. He, J. Z. Fu, and Z.C.Chen, "Temperature precise control in hot embossing device," Opt.Precis.Eng, vol 16, pp. 845-850, 2008.

[7] X. X. Li, W. Wang, and X.C.Chen, "Generalized predictivemultimode PID control for giant magnetostrictive actuators," Opt. Precis. Eng., vol. 18, pp. 412-419, 2010.

[8] P.C. Zhang, and Q. Zhang, "Research of the NTC Thermistors Equation of Calibration," Chinese J. Sensor Actuat, vol 25, pp.220223, 2012.

[9] X.Wu, Y. Ma, and Q.P. Dong, "Research on Real-time Operating System Performance Measurement," J. Syst. Simulat., vol 25, pp.313-316, 2013.

[10] Y.L.Vorokhovsky, S.V.Anastasyev, and A.A.Volkov, "A New Low Profile DOCXO". IEEE International Ultrasonics, Ferroelectrics, In: Frequency Control Joint $50^{\text {th }}$ Anniversary Conference, pp. 499-451, 2004

[11] T.Oita, M.Fukada, and A.Nakamura, "Dual Mode SC-Cut Crystal Oscillator," In: IEEE International Ultrasonics, Ferroelectrics, Frequency Control Joint 50th Anniversary Conference, pp. 436442, 2004.

[12] K. Rangaswamy, P. Sreekanth, and S.Sandu, "Fuzzy Fractional Order PID Based Parallel Cascade Control System," Adv. Intell. Syst. Comput., vol. 235, pp. 293-302, 2014.

[13] J.Yu, J.Zhuang, and D.H.Yu, "Fuzzy Anti-Windup Compensator Design with Applications," Chinese J. Sci. Instrum, vol. 47, pp. 6873, 2013.

[14] H.F.Nie, X.P.Li, and L.L.Zhang, "Temperature Control Algorithm for Optical Lithography Projection Lens," Chinese Mech. Eng., vol 19, pp. 1135-1139, 2008. 